\title{
INSUFICIENCIA CARDIACA CONGESTIVA POR ESTENOSIS PULMONAR CONGENITA
}

\author{
PRESEN'TACION DE, UN CASO \\ Lr. RLARCELO MOFFFANO

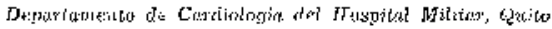

Con relativa frecucheia ss ubser. van cuadros de: inculfie:encia curntíuca angosiliva etr tuiñus menores du scis meses ds odad. Aiguners de etlos so

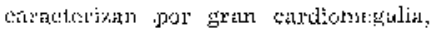

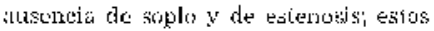

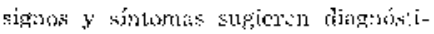
cos de mineardilis, 〔jbruelastosis, amilosclosis, naneimicato unómalı du pulmonares, etc.

Cuatudo apureseri formande us teu:

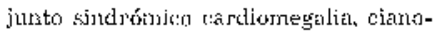
sis y presencia de soplo cartíato se suporto que son producto de cerdiopalito sever:a con pronóstico ǵteneril]mente mals.

I'resentatción de un maso.-La jiña N. P. rle 4 meses de edad fue exumineda por primera $v t:$ a la edad de tres

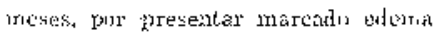
facioll $y$ de extromidades superiores interiores, sin aparesite compromijis eridínco o vaseufar centrit; fue ontontes referida, por el médico traínlle, Servicio re ]'olientria, con la jmpresió: djagnóstica de una mefropatia, tes vil'-

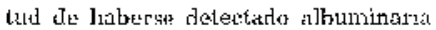
$+\cdots$ - en el cxamer correspordiente.

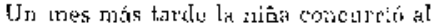
Depátamersto de Curdialougia con inurements dis edomis facial $y$ do extremidades, abombrmicrito abdominal progresivo, dusnejt exintinúa, marcada

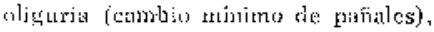
irr:tedbiliclad, evideule inorexia $y$ ius sor: serreniones resp:raicrias Jnóviles.

El examen lístco revoló una asitua de 16 libras de pesci, cot huenas pulsos

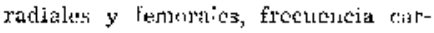

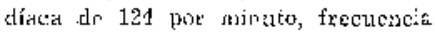
raspiratoria de 36 por ritintato.

Ldeme facial, de extrannidlades y ya-

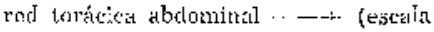
Le 0 a 4$)$, derrame astílico (1 - - ).

Prems!n loa cle rales de medianas burbujels en ambos sasnpos pulmonart:;, roncus y uedieuna rantistad de sib:lancias.

I.1epratumegulia can borde irferinr galpable a $5 \mathrm{~cm}$. debajo del rebolele (I)sial derecho.

El nxamer de corazól reveló: puntн

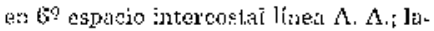

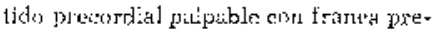
d々รminun: do ctaracteres nomales; $R_{2}$ intensidad 
nomilne, no se pudietor evidenciar in. dividualizaclos sus dos tomponentes. $\mathrm{R}_{3}$ attilble curnantemente en punta,

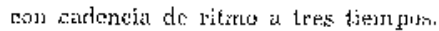
presencia de soplo punsistílicrs, intonvistad 3, mixime en punta, ircadistu difusimenté a iuda el árra procorcliat.

Exarnesy radialógico de corazún.Enorire lardioil:egy liy el ]a cua] llema lia alención la prominencia mareada se la auricula derecha ell ánleropustevjou, oun pusta cardiaca levantacle y di]atioción de arteria pulmonar (Fig. 1). La radjugraria ablicta anterior derechat proscutal un rerhizo posterisy do les siluet:a cerdíava "itmulando uma gram

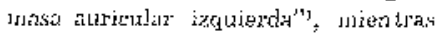
las si!netas ventricuias y antricular dereshitis contactan cxtensanente coln ] pared tostal interisur (1'jg. 2). La rarliografía oblícua anterior jzaduierda a vez presenta un arto ventrícular izafilertir dobordante y una prominencia ántero-superial situgerítiva de dilatación de la orojucla dercohi supexiruestą al arca de lil arteria pulmonar (Fig. 3).

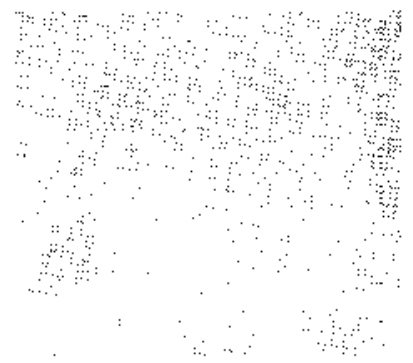

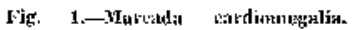
A frondamiento aluricular dejecho $y$ de irtexin puimonat.

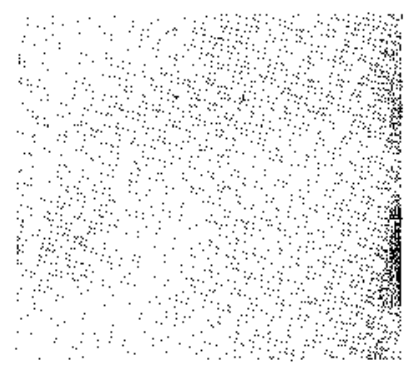

Jij. z_-Nótese la aparente ditylación

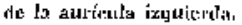

Fistudio electrotardiográíco- $\mathrm{No}$ íue yusib-e practicnúo clada la cortís:ma cvolusión que la pacionte tuw hajo ruestro cuidado, pues a posalr do ba terapia ionicardiaca, diurétice y sedanto la niña fallecis pors drepués de! $n$. greston.

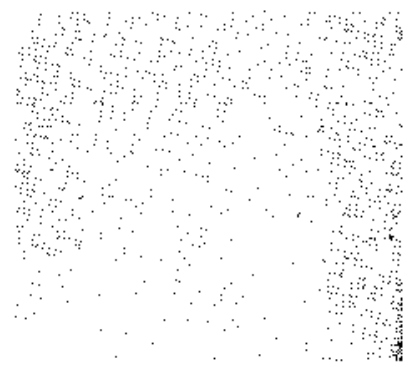

Iis. 3,-Nobese Ja aparents dilatarion dal ventriculn izquierelo $y$ de la turícula y arejuela dercesias.

Dxamon post-murten: 1) Cardiomegalia mas:va con rechazis posterior de ambusis pulmones, los ruales estaban pareialmexic solapsadns. 


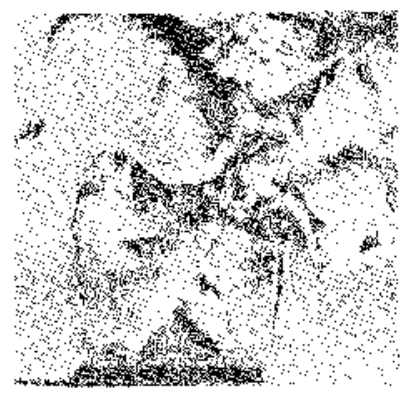

Fig. d.-Se apreciiz La miseata dilatación de la auriculu dexcełla ([lecta) y la marcada hiperiloofia de in pared yentricular deredta.

2) Por punción del saco perisárdico

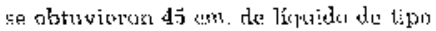
t.'asıldado, que si bien reducía el titmaňo de la cardioncenglia sisı umbarfu éta permanecía con caracteres l:amatives.

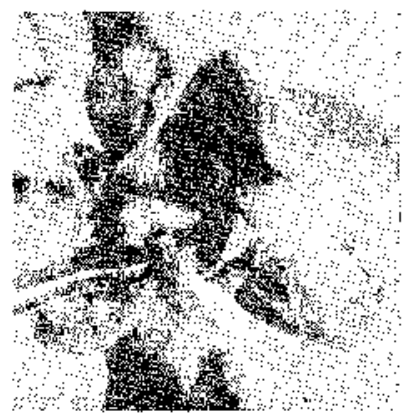

Fif, 5.-I demuestra van enorue envidid con steptum intcratricutar intacto.
3) Abierto el saco perícírdico pudo

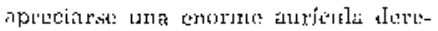
chn ( ${ }^{*}$ ig. 4), ]a misma clue al ser abier-

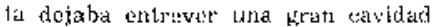
aurivalar con septu:n aurjeulitr integer (ivig. 5).

b) El septam interventricular era nisernat en su (rotrstiluxión, cumparliessdo de la marcada hipertrotia ventricular derecha.

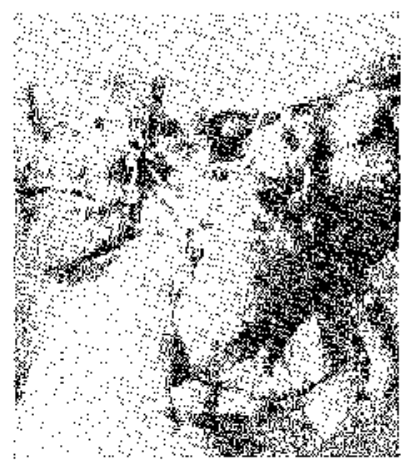

Fig. 6.-Is punta del seĩulador indiru cl arifieio puntifuremp de la pulunorar.

5) Lo vólvula tricúspide se meon-

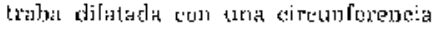
de 73 mm. Las paredes vantricularas derechas fueron marcidimente hipert.róficas, coul un espesor de 7 a 10 uוu, entre la xoma libece $y$ tribecelalada (Figsi. 4 y 5).

(i) Abiertu la arteria pilmonelr sn: encontró una estenoris valvular puntiforme, cuyo diánetro ex fresto be de

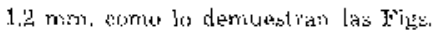
fi $y$. IL septum aórtieo pulnoner era normil. 


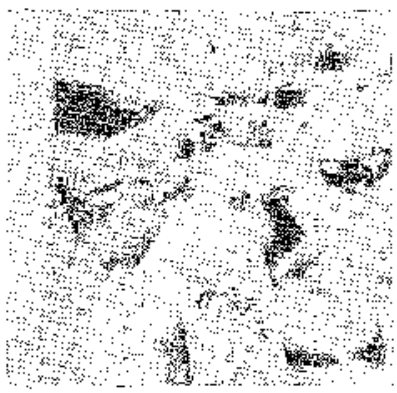

Fig. 7.-1,a arkeria pulmonax ahierts preasenta 1:a clásiata diliztiación puxtus-

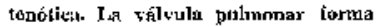

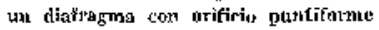
central.

7) El triayesto de la arteria pulnunax se hallaba tilatado cu el troncu priencipal, correspondiendo a la clisica dilatacjón post-estenólica. Sı virutufertecia mayor tue de $26 \mathrm{~mm}$.

8) J a uurícula $y$ ventriculo izquierdos cstuban reducidosi a dos cavi-

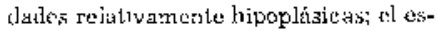
pesor de la pared ventricular izquierda fuo des 6 mm. La circunferencia mitral de $5.1 \mathrm{~mm}, y$ la circunferencia niayor de la aorta de $14 \mathrm{~mm}$.

\section{i)ISCUSION}

Va]crado el presente caso como el de una cardiopatia eon carrdiomegulia gigante, con insuficiencia cong estiva cs atlasiarea, con evolución clínica napíta, con sopley $y$ sin cianosis, la interprelaeión diagnóstice fue de insufieiencia mitral con comunicación inler-auricu-

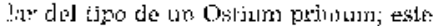
sritcrio se vé́a justif́jcado tanto pox las curicteristotis del soplo (pansistólico lo ponta con jrradiación difusa a lodo [] jrectrollo), cumo por los hatlazgos ladicogicos sugestivos de urs zgrandi-

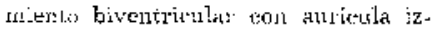
quirrdu gigante.

Por iss hellazos jotst-morton pusde dextucirse rue el derrame perichir-

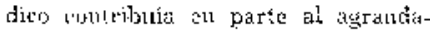
minsto enoxre dle la silueta cardíacia. La esternosis valv'zlar puntiforme de ia pulmonas cor intogridad de septursi ouricular $y$ ventrisu] hipolitufia ventrituliar dereoha que fue l:apaz dite mantener compenseslo al tro-

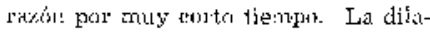
tariós posierior del ventriculo intuda-

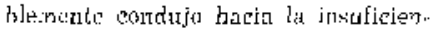
cia tricusiníldea, hiperteusión y luego desproporeionda dilatavicin de ausículit derocha e insuficiencia carthíata congestiva.

La iatlerptetación sumiológica aluc: origimalumente orisnto hacia el diagnóstice, de tena insuficientia mitral con Ostitu primutn, aratómicamente rese. ace un inecanismo explicativo tolalmente ditcrence, pues, la marcada rotación huraria del corelzón pröducida por la hipertrolid ventricular derecha hizn oue la insuficiencia tricuspidea fuera la causante del soplo sistílico de punta que permitió $1_{i l}$ confusión com instafieiencia mitral.

Nótese, asimismo, cue la siluela radrológica sugestiva de agrandamiento ventricular izquiexdo ni representa sino un marencio rechazo posterior de las cavidades ircuicrdas por dilatac:ón snasiva rle las derechas (Fig. 3). 
Ficta cardinpatía aengénito rexunece una incideneib bastarte baja, sthre l.odo on rolkicisia con la insuficiencize

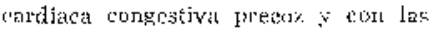

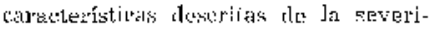

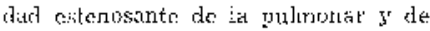
la difatación de lá zuriesda dorechil.

Segŕa varios autures a que har reavi sado este tema:-1-1, se dexprende cito aperiak $\mathrm{t}+\mathrm{l}$ 10\% de las esterusis pulmowares se unen a integrided absoluta ste saloipucs.

\section{RISLL:MLIN}

Fu una niňa de 4 neses, frịterids con signoä y sirti.umes atr! insuficsen:ia wadraca eongestiva, sc cncoutró al exilmen ryost-morten estemnsis pulanomar valvular puntiforme; de septum aurieular y vontrieslar que llevó a corto plazo hacia lis jusuficienciil ventritular derecha cor dilatación de válvula irjeúspide que semiológiceamente simuló una insuficiencia snitral; Jlama la atonciún la eqolución rupidisima rel cuaclro que ae enmprya como yna inmuficioncia cardiaca tonges:iva irreversible.

\section{SUMWARY}

A fisur months old girl died with signs and symptoms of congestive hearth fililuxe. At post-Inuxtom examination, a pin-pointed valvular pulmonary stenosis, with nu domage whatsoever at auricular or ventrieular scp$1.11 \mathrm{n}$, was lourd. This condition broidght in a very shout interver? right ventricu-

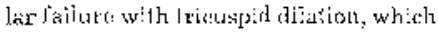
simulited mitral sosufficiency. The strikingly ratek oliniten] evolution ol 1.) i:; cass, which hat the belnavion of: im- irrerersible tungestive herd lit:lure, is minst wolthy af attention.

\section{RLLLIENCIAS BIBLIOGRAFICAS}

1.-K.iELBLdea, S. R.: Diakinosiz of eongenital hourt discuec, 123, 1055.

2.-W96, 23: Cnngenital heart discase. A review of its clinieal aspects in tin likht of expczituce rained by trasus of muderat eckmiques. Brit, Mer, J. 2: k.14, 195n.

3. -TAIJSSING, FI. B.: Congonital mäformutions of the hearl. Kow York Commonwevalth Futud, 1947.

1. NADAS, A.: Pediatric endjolony. Surtder: Cu., 4it6, 1957. 